\title{
A New Model of a 3D-Printed Shell with Convex Drug Release Profile
}

Jingzhou Zhao ${ }^{1}$, Xiaowen $\mathrm{Xu}^{{ }^{1}}{ }^{*}$, Maonan Wang ${ }^{1}$, Liang Wang ${ }^{2}$

${ }^{1}$ Central South University, Changsha 410083, China

${ }^{2}$ School of Medical Informatics, Xuzhou Medical University, Xuzhou, Jiangsu, 221000, China

\section{ABSTRACT}

e-mail: xuxiaowen@csu.edu.cn

The objective of this study was to fabricate a tablet with a convex drug release profile. A circumscribed sphere with a regular tetrahedron (pyramid) cavity shell model was created by a computer-aided design (CAD) program and printed by a 3D printer. Paracetamol (APAP) mixed with polyvinyl alcohol (PVA) gel was filled into the cavity. The tablets were obtained through drying. The release profile was obtained by detecting the APAP concentration in phosphate buffer solution through ultraviolet absorption spectrophotometry. Along with the decrement of tablet diameter, drug exposure area gradually increases the release rate of the drug. The results indicate that the release profile from a 3D-printed PVA shell with a tetrahedron cavity is increasing with no residue after dissolution. Potential fields of application may include hypotensors and other types of personalized medicines. Further studies are necessary to explore the potential application of more structures.

KEYWORDS: 3D printing, controlled release, polyvinyl alcohol (PVA), regular tetrahedron, dissolution

\section{INTRODUCTION}

Ther large-scale drug production is undoubtedly beneficial to human beings; however, there are limitations in the traditional medicine industry. The degree of customization is not high enough to meet the individual needs of all patients. Therefore, a great breakthrough is highly desired in the pharmaceutical industry. 3D printing (3DP) technology was invented in the late 1980s and spread quickly throughout almost all manufacturing industries including medicinal manufacturing because of its customizability. In August 2015, the US Food and Drug Administration (FDA) approved the first global 3D printer to be applied in the pharmaceutical market (1).

Controlled dissolution of medicine provides convenience to patients. Compared with other manufacturing methods in pharmaceutical companies, 3DP of fused deposition modeling (FDM) has some incomparable advantages. First, it reduces the time of turning an idea into a product, which makes customized products more quickly. Secondly, it can construct complex hollow structures easily. Finally, it has cost effectiveness. These advantages make 3DP-based controlled-release drugs highly attractive and easily produced (2).

*Corresponding author.
After years of development, 3DP technology currently consists of several major sub-categories including FDM, stereo lithography apparatus (SLA), selected laser sintering (SLS), laminated object manufacturing (LOM), continuous liquid interface production (CLIP), and powder based (PB). Among these techniques, FDM, SLA, SLS and $\mathrm{PB}$ are commonly used in medicine manufacturing $(3,4,5)$. Most of all, FDM is one of the most regularly used low-priced methods in 3DP (2). The retail price of a desktop FDM 3D printer can be as cheap as $¥ 800$ (\$124 US) in China. 3DP is established for non-pharmaceutical aims in a broad scope of low-cost printing application (6). This type uses a heated head to melt raw materials, and utilizes a thread extruder to extrude organic filaments [e.g. ABS (acrylonitrile butadiene styrene), PLA (polylactic acid), etc.]. When printing a product, the 3D printer deposits melted filaments through a predetermined path, layer by layer, to solidify them in several seconds. Furthermore, the tablets made with FDM 3DP are more uniform and stable (7).

Medical researchers use FDM 3DP to fabricate certain tablet forms. In fact, several techniques are developed by researchers with the aim of constructing tablets that release drug ingredients with specific profiles such as convex, bimodal, or linear profiles (8). Goyanes et al. 
mixed paracetamol (APAP) into polyvinyl alcohol (PVA) powder, made filaments, and then printed out five different geometric shapes with equal weight, surface area, and surface area-to-volume ratio. The APAP-release reaction reveals that surface area-to-volume ratio is a determinant of drug release profiles (9). 3D-printed implants have micro structures with complex dissolution profiles (10). The most impressive function of 3DP is the complex generation of drug structure. What's more, its release profile could be designed to control the release rates of drugs (11).

In a recent study, researchers have created a 3D-printed template to produce a specialized tablet with customized drug release profiles (8). Although the target is attractive, this method shows an apparent disadvantage: as a multistep process, it is too sophisticated to be applied to massive manufacturing. In addition, the materials it needs are hard to be collected. Another major limitation for the application of FDM is elevated melting temperature (around $220^{\circ} \mathrm{C}$ ) required for its implants, which would seriously degrade the content of pharmaceutical excipients and active components (12).

In this study, a new method is reported to control medicine release profiles. First, a specialized shell with an external round shape and internal regular tetrahedron (pyramid) shape is fabricated by 3DP, which allows injected medicine to form a tetrahedral shape in the cavity. When the shell is dissolved, the drugs are gradually exposed to buffer solution. The tablets will be dissolved together with the shell from its four corners. The surface area of the drugs will gradually increase the ratio of it to the shrinking diameter of the tablets (Fig. 1). The change of surface area will also facilitate the release rates of the drugs. This kind of shell can be easily manufactured by 3DP technology and can obtain convex drug release profiles. Injecting medicine into the cavity avoids hightemperature heating and is more suitable for the heatsensitive ingredients. Convex drug release profiles are suitable for some special purposes. For example, the peak blood pressure in patients with hypertension generally occurs from 6 a.m. to 10 a.m. (13), but it is hard to comply with the medical advice of taking hypotensors before 6 a.m. or much earlier than that every day. Thus, a customized hypo-tensor is needed that would allow patients to take their medicine at night and have blood concentration reach its maximum in the morning. With our model, peak blood concentration will be delayed for $7 \mathrm{~h}$ after the medicine is taken.

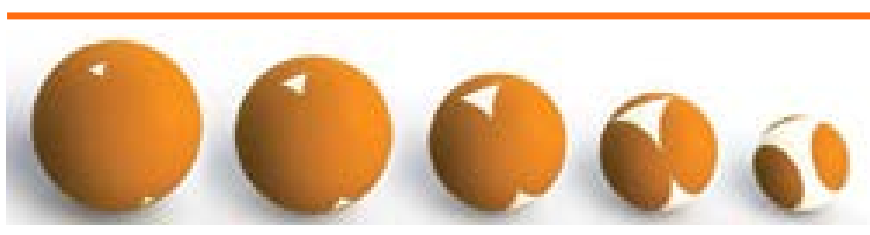

Figure 1. Model of controlled release tablet. The white part is the inner medicine core; the orange part is the outside shell. Along with the decrease of tablet diameter, the release rate of the medicine gradually increases.

\section{MATERIAL AND METHODS}

PVA (molecular formula $\left.\left(\mathrm{C}_{2} \mathrm{H}_{4} \mathrm{O}\right)_{n}\right)$, (YingJia Inc., China) was used for 3DP. PVA filament (DFRobot Inc., China) diameter was $1.75 \mathrm{~mm}$. Temperature of the printing process ranged from $180-200^{\circ} \mathrm{C}$, which was available in most types of FDM 3D printers.

\section{Preparing Gel with APAP and PVA}

PVA powder (4 g) and APAP powder (2 g, Anta Biotechnology, China) were added to $4 \mathrm{~mL}$ of water and mixed thoroughly.

\section{Building a Circumscribing Shell Model}

A circumscribing sphere model with a regular tetrahedron cavity was set up by computer aided design (CAD). A regular tetrahedron was added into the sphere by making sure that the geometric centers of the two shapes coincide. The Boolean operation was performed to get the complete sphere model with a hollow tetrahedron cavity (Fig. 2).

\section{Printing the Shell}

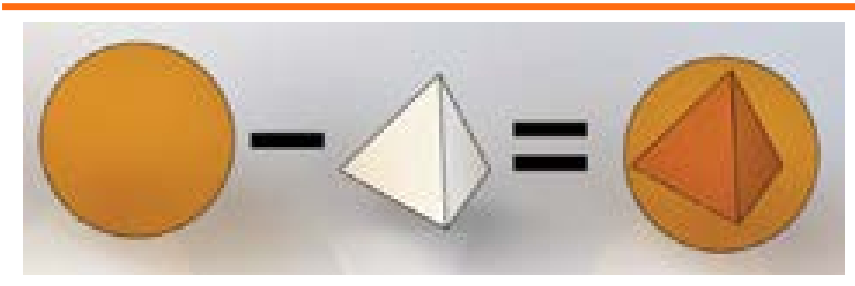

Figure 2. Building a circumscribed shell model. Diameter of circumscribed sphere is $12.4 \mathrm{~mm}$; Side length of tetrahedron (pyramid) is $10 \mathrm{~mm}$.

The 3D printer used in this study was a standard FDM printer (Creator Pro, FlashForge, China). The shell model is loaded in Slic3r software (version 1.2.9), and printer settings are as follows: temperature $\left(180^{\circ} \mathrm{C}\right)$, layer height $(0.15 \mathrm{~mm})$, number of shells (2), infill rate $(100 \%)$, nozzle diameter $(0.3 \mathrm{~mm})$, and print speed $(80 \mathrm{~mm} / \mathrm{s})$.

\section{Injecting Gel into PVA Shell}

First, a small hole with a diameter of $0.7 \mathrm{~mm}$ was drilled at the thinnest part of PVA shell. Prepared gel was injected into the regular tetrahedron cavity by a 1-mL syringe 
with a needle (inner diameter of $0.5 \mathrm{~mm}$ ) from the hole of the shell (Fig. 3). The average weight of each PVA shell before and after injecting was $900 \mathrm{mg}$ and about 1000 $\mathrm{mg}$, respectively. The tablets were put into a drying oven at $70{ }^{\circ} \mathrm{C}$ for 8.5 hours (Fig. 4B). Tests were conducted in triplicate under sink condition, and each tablet contained approximately $20 \mathrm{mg}$ of APAP. The diameter of the circumscribing sphere was about $12 \mathrm{~mm}$.

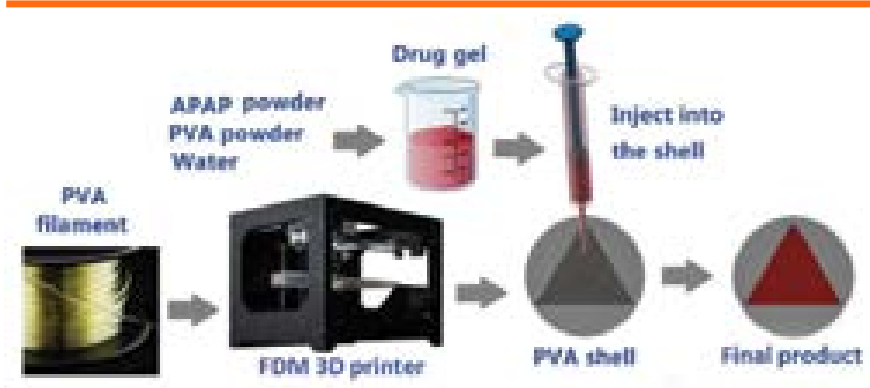

Figure 3. The process of fabricating tablets. PVA, polyvinyl alcohol; APAP, paracetamol.

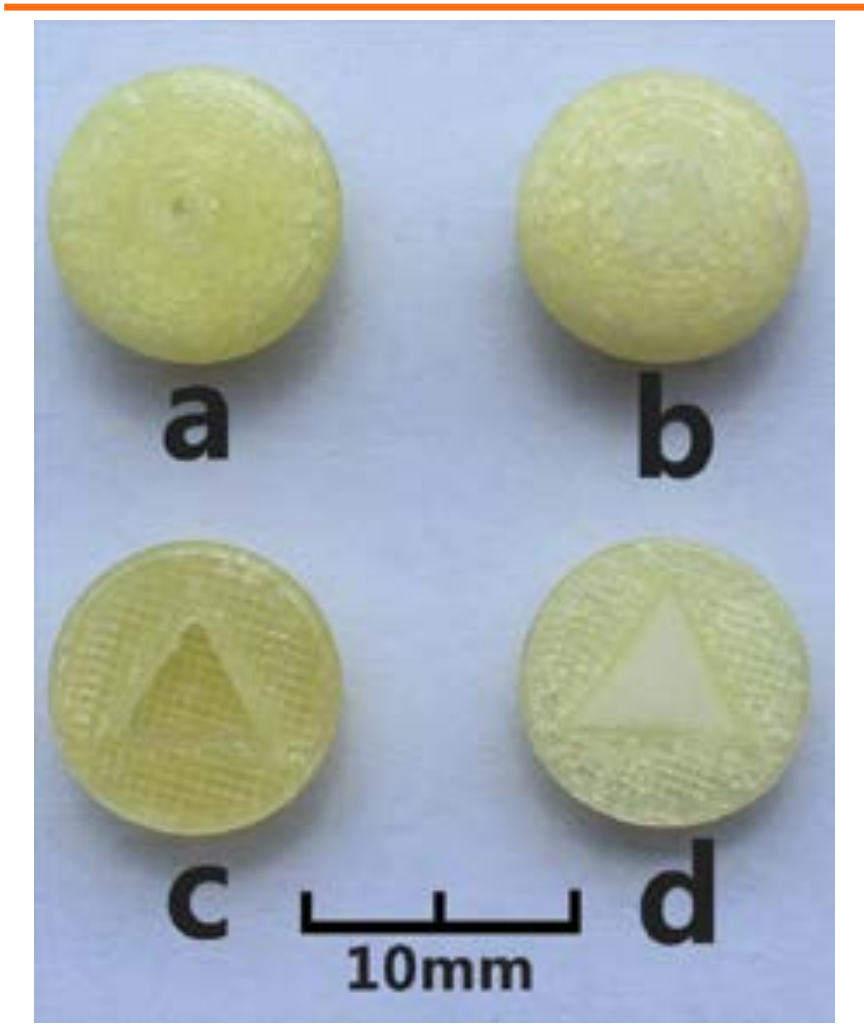

Figure 4. The form of the constructed tablet. (a) PVA shell with a hole; (b) shell injected with drugs; (c) cross section of shell before injection; (d) cross section of shell after injection.

\section{Dissolution Testing}

Drug release rate was measured in time series with a USP type II apparatus. Each tablet was put into $900 \mathrm{~mL}$ of phosphate buffer solution (PBS, $\mathrm{pH}=6.8$ ). The buffer had a better resemblance to the characteristics of gastrointestinal fluid in terms of $\mathrm{pH}$. The buffer solution was continuously heated and stirred $(50 \mathrm{rpm})$ at $37^{\circ} \mathrm{C}$ for about 8 hours. Samples were taken out every 15 minutes and kept standing for 15 minutes before measurement. After each sample was measured, the sample $(5 \mathrm{~mL})$ was returned to the solution again. The absorption of each sample was measured by UV spectrophotometry (UV2601, Benjing Beifen-RuiLi Analytical Instrument (Group) Co., Ltd, China)

The absorption of APAP buffer solution at $243 \mathrm{~nm}$ was detected before the samples were tested to suppress interference. In addition, this experiment needed a dual wavelength measurement to alleviate the impact of PVA absorption because there was a superposition between PVA absorption and APAP absorption. After several experiments, another wavelength of $264 \mathrm{~nm}$ was selected to eliminate the impact of PVA on APAP. Each sample was detected at $243 \mathrm{~nm}$ and $264 \mathrm{~nm}$ respectively, so an average value was calculated after multiple measurements. Moreover, measurement results were the superposition values of APAP and PVA (Eq. (1) and (2)):

$\mathrm{Abs}_{243}=A \mathrm{As}_{(\mathrm{APAP}) 243}+\mathrm{Abs}_{(\mathrm{PVA}) 243}$

$\mathrm{Abs}_{264}=\mathrm{Abs}_{(\mathrm{APAP}) 264}+\mathrm{Abs}_{(\mathrm{PVA}) 264}$

where Abs is absorbency.

However, 243 and $264 \mathrm{~nm}$ determined the slope of the absorbency-concentration curves of $\varepsilon(243)$ and $\varepsilon(264)$, respectively. The calculated $\Delta \varepsilon=\varepsilon(243)-\varepsilon(264) ; \varepsilon(243)$ and $\varepsilon(264)$ were 15.290 and 6.485 in dissolution testing, while the absorbance of PVA was the same in 243 and $264 \mathrm{~nm}$. Equation (1) - (2) was the concentration of APAP (Eq. 3):

$$
\begin{aligned}
& \mathrm{Abs}_{243}-\mathrm{Abs}_{264}=A \mathrm{As}_{(\mathrm{APAP}) 243}-\mathrm{Abs}_{(\mathrm{APAP}) 264}= \\
& (\varepsilon(243)-\varepsilon(264)) \mathrm{bc}
\end{aligned}
$$

where $b$ is a constant $1 \mathrm{~cm}$ and $c$ is the concentration.

\section{RESULTS AND DISCUSSION}

The aim of this study is to fabricate a high-strength and high-stability tablet with convex drug release profiles. It possesses a complex shell structure printed by a FDM 3D printer. As shown in Figs. 4 and 5, the core of the shell is filled with corresponding medicine that adheres closely to the inner wall of the shell, even when it is dried and hardened. There are no obvious bubbles in the two parts of the tablets (Fig. 5), indicating that the injection is 
feasible. The drugs are relatively firm as a whole in terms of physical strength; hence, it is not easy to collapse. This is consistent with the results of many studies of PVA 3D printed drugs (2).

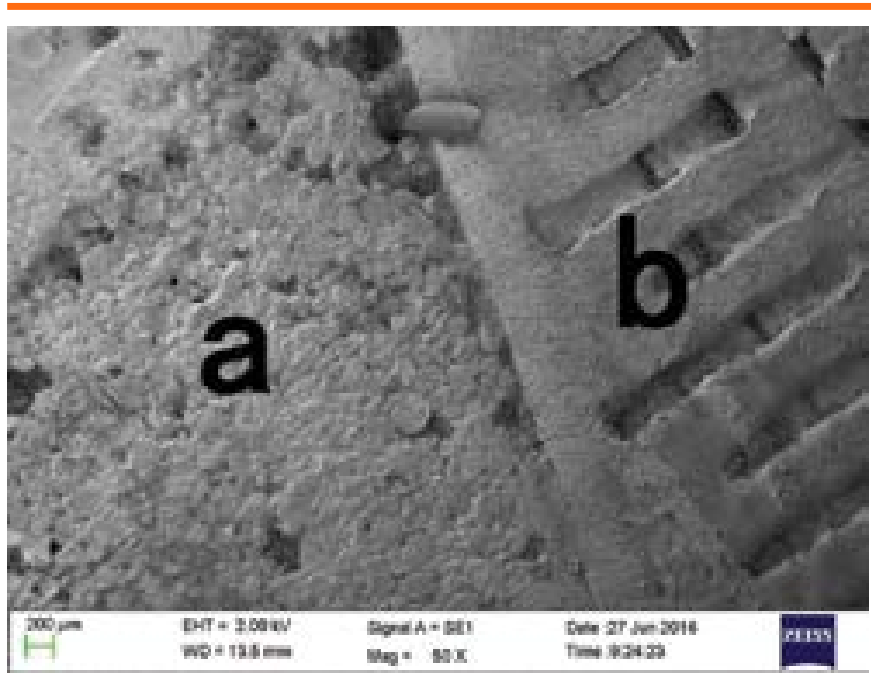

Figure 5. Scanning electron microscope (SEM) image of cross section of (a) drug gel after injecting and drying and (b) PVA shell printed by FDM printer (cross section is milled by sandpaper).

Our drugs include an increasing release profile that is important for patients who have developed tolerance to a drug in medication (8). To test whether our model can gradually control increasing medicine release rate, the tablets are dissolved in PBS buffer and APAP concentration is detected every $15 \mathrm{~min}$ with ultraviolet absorption spectrophotometry. As expected, APAP concentration is maintained steadily at a low level at the beginning (from 0 to $300 \mathrm{~min}$ ), then increases rapidly after $300 \mathrm{~min}(5 \mathrm{~h})$, and finally reaches peak value at $450 \mathrm{~min}$ (7.5 h) (Fig. 6). Goyanes et al. printed APAP with PVA and studied the influence factors of medicine dissolution rate (9). Their results indicate that the dissolution rate of the PVA tablet varies with surface area, weight, and volume. However, no matter what shape, mass, or volume a single component of the drug is, its release profile is always decreasing, because the area of active ingredients is not controlled. In our study, the increasing release profile depends on a regular tetrahedron, indicating that the change of exposed surface area will significantly affect the release rate of the drugs. It is difficult to predict the change of available area accurately, because the relationship between radius and time is not linear. The dissolution rates of PVA gel and APAP gel are almost the same. It is believed that an erosion-mediated process drives drug dissolution, and the dissolution profile seems to end at a higher concentration than expected in $900 \mathrm{~mL}$ of buffer with $20 \mathrm{mg}$ of drug. To our knowledge, evaporation may cause the concentration to be slightly higher at about 8 hours and has little impact on the results.

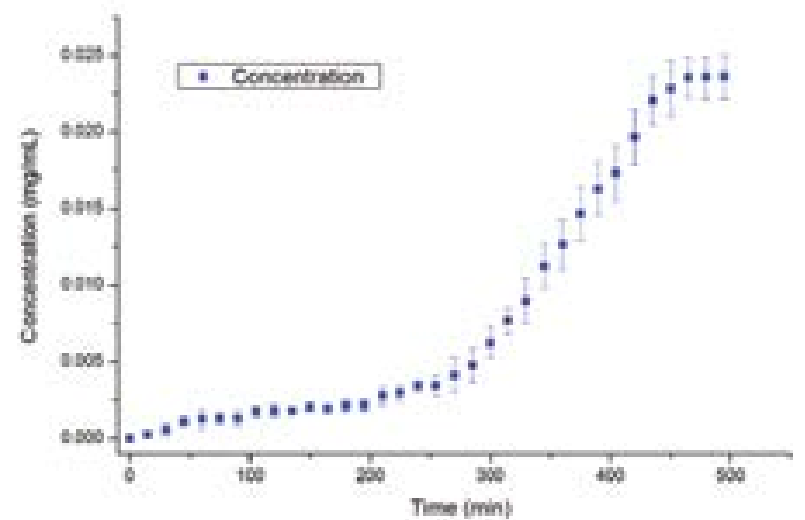

Figure 6. Paracetamol (APAP) release profile of the tested tablet. Error bars represent replicate dose units.

A highly stable drug release profile with no residual is important. To control the drug release profile, an insoluble container with one hole was used (8). This kind of structure would be easily affected by impurities or gastrointestinal peristalsis. Our model shows simultaneous dissolution of the core and shell of tablets in solubility tests, with uniform distribution of four curved line triangles on the sphere shell. This kind of geometry is not susceptible to interference by other factors during release. In the production process, each tablet is loaded with $21 \mathrm{mg}$ of APAP.

The FDM 3D printer needs a high temperature to melt filaments, inevitably influencing or even damaging the active ingredients of the drugs. In this study, the drugs were dissolved into gel and injected into the cavity of a printed shell, thereby avoiding being heated to the melting temperature and protecting the active ingredients of the drugs to the utmost extent (14). Our model can be widely applied to temperature-sensitive drugs. Theoretically, any drug that does not react with PVA can be injected into the cavity of the 3D-printed model.

3DP geometric cavity entities have strong operability and give full play to the potential of 3DP technology. The disadvantage is that the printed and molded drug shells can only be used after injecting and drying. In addition, the drug loading capacity of the tablets could only reach to $2 \%$. In a recent study, a filament extruder has approximately $4 \%$ of APAP loaded into PAV filaments, 
which are suitable use in a fused deposition 3DP model. 3DP may not be suitable for higher volume production (9).

Our work has shown the potential of 3DP technology to manufacture personalized medicine. In further work, we intend to use dual head printing. High-temperature materials (PVA) and room temperature materials (gel) will be printed together with two switchable nozzles, which can reduce the volume of tablets and improve drug content. The next stage of our study will focus on extrusion-based (EXT) printing, because EXT has a drug loading capacity of up to $90 \%(15)$.

\section{CONCLUSION}

Our 3D-printed shell model of a circumscribed sphere with a regular tetrahedron cavity obtained a convex drug release profile. The dissolution rate increased with decreasing tablet diameter, and PVA material dissolved with no residue. Other cavity shapes, such as cube and rectangular, and two or more cavities may also induce different drug release profiles with distinct attributes that have high potential for future applications.

\section{ACKNOWLEDGMENTS}

This study was supported by National Natural Science Foundation of China (No. 21105127) and Fundamental Research Funds for the Central Universities of Central South University (No. 2017zzts389) and the medical electronics MakerSpace of Central South University.

\section{CONFLICT OF INTEREST}

The authors declare no funding or conflicts of interest related to this article.

\section{REFERENCES}

1. Norman, J.; Madurawe, R. D.; Moore, C. M. V.; Khan, M. A.; Khairuzzaman, A., A new chapter in pharmaceutical manufacturing: 3D-printed drug products. Adv. Drug Del. Rev. 2017, 108, 39-50. DOI: 10.1016/j.addr.2016.03.001.

2. Alhnan, M. A.; Okwuosa, T. C.; Sadia, M.; Wan, K-W.; Ahmed, W.; Arafat, B., Emergence of 3D printed dosage forms: opportunities and challenges. Pharm. Res. 2016, 33, 1817-1832. DOI:10.1007/ s11095-016-1933-1.

3. Wang, J.; Goyanes, A.; Gaisford, S.; Basit, A. W., Stereolithographic (SLA) 3D printing of oral modified-release dosage forms. Int. J. Pharm. 2016, 503, 207-212. DOI: 10.1016/j.ijpharm.2016.03.016.

4. Ayala, G. E.; Dai, H.; Powell, M.; Li, R.; Ding, Y.; Wheeler, T. M.; Shine, D.; Kadmon, D.; Thompson, T.; Miles, B. J.; Ittmann, M.
M.; Rowley, D., Cancer-related axonogenesis and neurogenesis in prostate cancer. Clin. Cancer Res. 2008, 14, 7593-7603. DOI: 10.1158/1078-0432.ccr-08-1164.

5. Meléndez, P. A.; Kane, K. M.; Ashvar, C. S.; Albrecht, M.; Smith, P. A., Thermal inkjet application in the preparation of oral dosage forms: dispensing of prednisolone solutions and polymorphic characterization by solid-state spectroscopic techniques. J. Pharm. Sci. 2008, 97, 2619-2636. DOI: 10.1002/jps.21189.

6. Wong, K. V.; Hernandez, A., A review of additive manufacturing. ISRN Mech. Eng. 2012, 208760. DOI: 10.5402/2012/208760.

7. Skowyra, J.; Pietrzak, K.; Alhnan, M. A., Fabrication of extendedrelease patient-tailored prednisolone tablets via fused deposition modelling (FDM) 3D printing. Eur. J. Pharm. Sci. 2015, 68, 11-17. DOI: 10.1016/j.ejps.2014.11.009.

8. Sun, Y.; Soh, S., Printing tablets with fully customizable release profiles for personalized medicine. Adv. Mat. 2015, 27, 78477853. DOI: 10.1002/adma.201504122.

9. Goyanes, A.; Robles Martinez, P.; Buanz, A.; Basit, A. W.; Gaisford, S., Effect of geometry on drug release from 3D printed tablets. Int. J. Pharm. 2015, 494, 657-663. DOI: 10.1016/j. ijpharm.2015.04.069.

10. Habibovic, P.; Gbureck, U.; Doillon, C. J.; Bassett, D. C.; van Blitterswijk, C. A.; Barralet, J. E., Osteoconduction and osteoinduction of low-temperature 3D printed bioceramic implants. Biomaterials 2008, 29, 944-953. DOI: 10.1016/j. biomaterials.2007.10.023.

11. Khaled, S. A.; Burley, J. C.; Alexander, M. R.; Yang, J.; Roberts, C. J., 3D printing of tablets containing multiple drugs with defined release profiles. Int. J. Pharm. 2015, 494, 643-650. DOI: 10.1016/j. ijpharm.2015.07.067.

12. Goyanes, A.; Chang, H.; Sedough, D.; Hatton, G. B.; Wang, J.; Buanz, A.; Gaisford, S.; Basit, A. W., Fabrication of controlledrelease budesonide tablets via desktop (FDM) 3D printing. Int. J. Pharm. 2015, 496, 414-420. DOI: 10.1016/j.ijpharm.2015.10.039.

13. Marfella, R.; Gualdiero, P.; Siniscalchi, M.; Carusone, C.; Verza, M.; Marzano, S.; Esposito, K.; Giugliano, D., Morning blood pressure peak, QT Intervals, and sympathetic activity in hypertensive patients. Hypertension 2003, 41, 237-243. DOI: 10.1161/01. hyp.0000050651.96345.0e.

14. Sandler, N.; Salmela, I.; Fallarero, A.; Rosling, A.; Khajeheian, M.; Kolakovic, R.; Genina, N.; Nyman, J.; Vuorela, P., Towards fabrication of 3D printed medical devices to prevent biofilm formation. Int. J. Pharm. 2014, 459, 62-64. DOI: 10.1016/j. ijpharm.2013.11.001.

15. Khaled, S. A.; Burley, J. C.; Alexander, M. R.; Yang, J.; Roberts, C. J., $3 \mathrm{D}$ printing of five-in-one dose combination polypill with defined immediate and sustained release profiles. J. Control. Rel. 2015, 217, 308-314. DOI: 10.1016/j.jconrel.2015.09.028. 